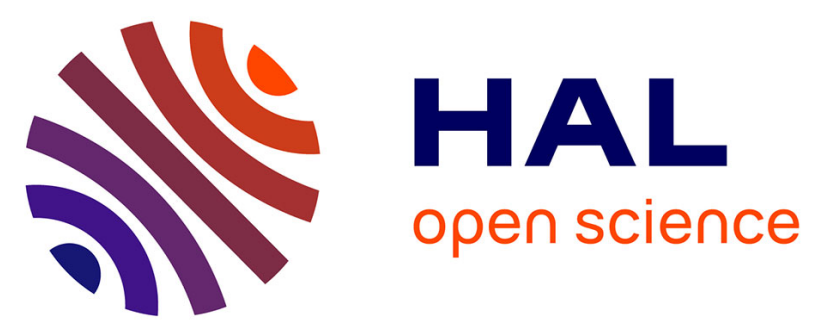

\title{
Limitation fondamentale dans les transistors MOS de puissance; le compromis entre la résistance à l'état passant RON et la tension de claquage VDBR
}

\author{
P. Rossel, Henri Tranduc, M. Gamboa, T. Phan Pham
}

\section{- To cite this version:}

P. Rossel, Henri Tranduc, M. Gamboa, T. Phan Pham. Limitation fondamentale dans les transistors MOS de puissance; le compromis entre la résistance à l'état passant RON et la tension de claquage VDBR. Revue de Physique Appliquée, 1981, 16 (9), pp.509-515. 10.1051/rphysap:01981001609050900 . jpa-00244943

\section{HAL Id: jpa-00244943 https://hal.science/jpa-00244943}

Submitted on 1 Jan 1981

HAL is a multi-disciplinary open access archive for the deposit and dissemination of scientific research documents, whether they are published or not. The documents may come from teaching and research institutions in France or abroad, or from public or private research centers.
L'archive ouverte pluridisciplinaire HAL, est destinée au dépôt et à la diffusion de documents scientifiques de niveau recherche, publiés ou non, émanant des établissements d'enseignement et de recherche français ou étrangers, des laboratoires publics ou privés. 


\title{
Limitation fondamentale dans les transistors MOS de puissance ; le compromis entre la résistance à l'état passant $\boldsymbol{R}_{\mathrm{ON}}$ et la tension de claquage $V_{\text {DBR }}$
}

\author{
P. Rossel, H. Tranduc, M. Gamboa et T. Phan Pham \\ Laboratoire d'Automatique et d'Analyse des Systèmes, 7, avenue du Colonel-Roche, 31400 Toulouse, France
}

(Reçu le 30 mars 1981, révisé le 10 juin 1981, accepté le 12 juin 1981)

\begin{abstract}
Résumé. - On définit les limites théoriques des variations du produit résistance $O N$-surface en fonction de la tension de claquage $V_{\mathrm{DBR}}$ dans les transistors MOS de puissance : Pour les basses tensions $\left(V_{\mathrm{DBR}}<200 \mathrm{~V}\right)$, ce produit $\left[R_{\mathrm{ON}} . S\right]$ est essentiellement imposé par le produit de la longueur de canal par la distance séparant les cellules élémentaires de diffusion de source et il ne dépend pas de la tension de claquage. En régime de hautes tensions $\left(V_{\mathrm{DBR}}>200 \mathrm{~V}\right)$ ce produit suit un mode d'évolution proportionnel à $V_{\mathrm{DBR}}^{-2,5}$ et ne dépend pas de la géométrie. Il apparaît enfin que les performances actuelles des structures D.MOS ou V.MOS sont inférieures d'un facteur voisin de 3 aux limites théoriques.
\end{abstract}

\begin{abstract}
The theoretical limitations of the product - ON Resistance-Breakdown voltage - are determined for the power MOST family. In the low voltage case $\left(V_{\mathrm{DBR}}<200 \mathrm{~V}\right)$ this product is proportionnal to the channel length and to the distance between the elementary source cells. It is independent of the breakdown voltage value. In the high voltage case, this product is proportionnal to $V_{\mathrm{DBR}}^{-2.5}$ and is not affected by the geometry of the device. The state of the art for D. and V.MOS, is one third of the theoretical limits.
\end{abstract}

Introduction. - Depuis 1968, de nombreux prototypes de transistors MOS dits de puissance ont été réalisés sous la forme de structures coplanaires à canal horizontal (D.MOS) ou de structures non coplanaires à canal horizontal (D.MOS) ou vertical (V.MOS, U.MOS), ces transistors à canal court présentent tous une structure multicouche : source $\mathrm{N}^{+}$, zone de canal $\mathrm{P}$, zone dite de drift $\mathrm{N}^{-}$et drain $\mathrm{N}^{+}$. Par ailleurs la configuration géométrique des petites cellules constitutives des transistors MOS élémentaires, qui sont mises en parallèle pour réaliser le transistor de puissance, diffère suivant le réalisateur du transistor; elle peut être hexagonale (HEXFET [1]), carrée (T.MOS [2], SIP MOS [3]), rectangulaire ou linéaire (EPIFET [4], V.MOS [5], U.MOS [6]). Enfin les profils de dopage entre source et drain varient d'une structure à l'autre sans que les motivations liées à ces différences soient clairement exprimées. En fait en se rappelant qu'un composant de puissance doit être à la fois capable de supporter des tensions élevées dans l'état bloqué, et de présenter une faible chute de tension à ses bornes lorsqu'il transite des courants importants dans l'état passant, le critère de qualité le plus important, élément majeur de comparaison entre les divers composants de puissance, est la carac- téristique - tension de claquage $V_{\mathrm{DBR}}$, résistance dans l'état passant $R_{\mathrm{ON}}$. Dans cet article, nous nous proposons de définir la caractéristique limite $R_{\mathrm{ON}}\left(V_{\mathrm{DBR}}\right)$ pour les transistors MOS de puissance. Pour ce faire, dans un premier temps, nous analyserons les effets de la contribution du canal du transistor et celle de la zone de drift $\mathrm{N}^{-}$à la valeur de la résistance $R_{\mathrm{ON}}$ puis calculerons la caractéristique $R_{\mathrm{ON}}^{-1}$ par unité de surface de silicium en fonction de la tension de claquage $V_{\mathrm{DBR}}$.

1. La résistance à l'état passant $\boldsymbol{R}_{\mathrm{ON}}$ - Nous définissons cette résistance comme étant l'inverse de la pente de la caractéristique de sortie - courant drain $I_{\mathrm{D}}$, tension drain $V_{\mathrm{D}}-$ lorsque la tension drain-source tend vers la valeur nulle. La figure 1 montre l'allure d'un réseau de caractéristique de sortie $I_{\mathrm{D}}\left(V_{\mathrm{D}}\right)$ au voisinage de l'origine. Sur la figure 2 , les valeurs de la résistance $R_{O \text {, }}$ sont reportées en fonction de la tension de grille; il apparaît que $R_{\mathrm{ON}}$ diminue avant de saturer lorsque la tension $V_{G}$ augmente, et tend vers une valeur que nous appellerons $R_{\mathrm{ON} x}$.

Cette résistance $R_{O}$, peut être décomposée en trois résistances [7] comme nous l'indiquons schéma- 


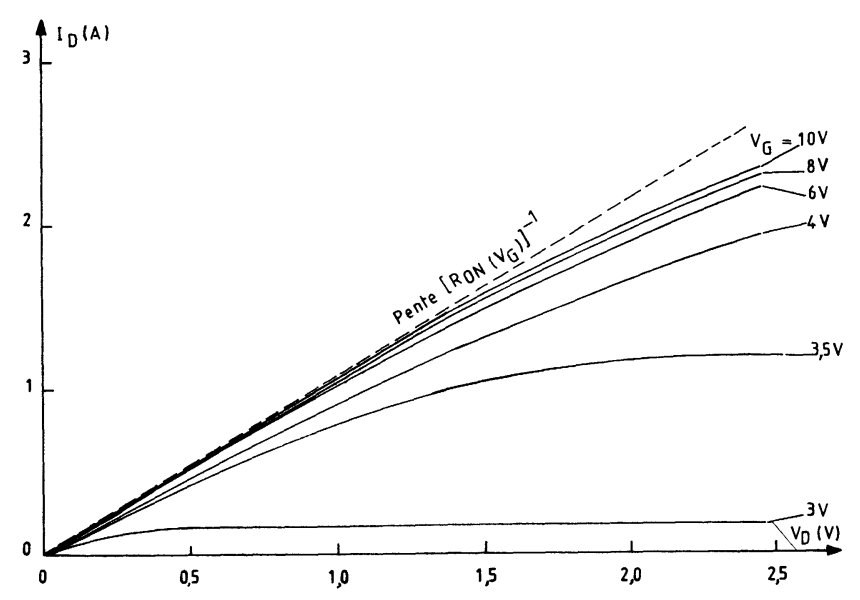

Fig. 1. - Définition de la résistance $\boldsymbol{R}_{\mathrm{ON}}$ sur le réseau des caractéristiques de sortie (transistor SIPMOS, MOS N, $16 \mathrm{~mm}^{2}, 250 \mathrm{~V}$ ).

[Definition of the ON-resistance.]

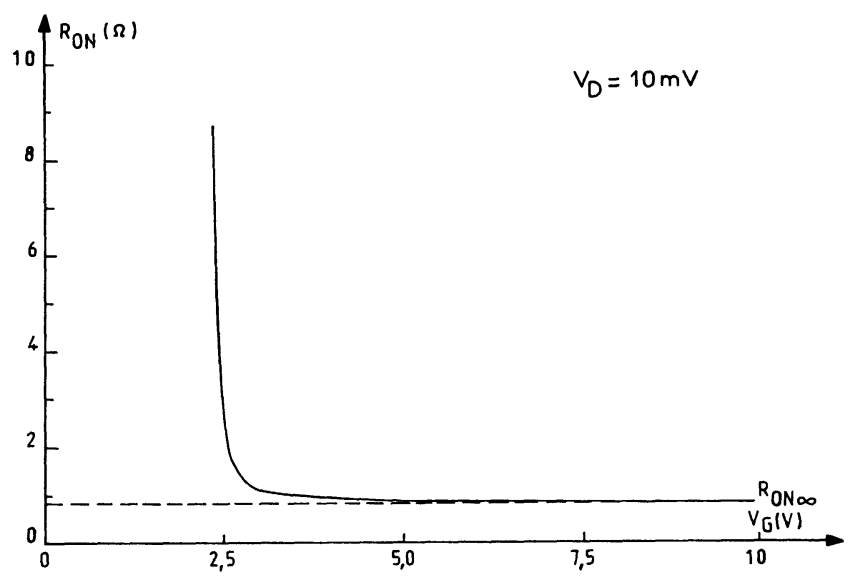

Fig. 2. - Variations de la résistance $R_{\mathrm{ON}}$ avec la tension de grille et définition de $R_{\mathrm{ON} \infty}$ (transistor SIPMOS, MOS N, $16 \mathrm{~mm}^{2}$, $250 \mathrm{~V})$.

[Variations of the $\mathrm{ON}$-resistance as a function of the gate-source voltage. Definition of $R_{\mathrm{ON} \infty}$.]

tiquement sur la figure 3 qui représente la coupe d'une des cellules élémentaires qui forment ensemble le transistor MOS de puissance. La première est la résistance $R_{\mathrm{ch}}$ du canal d'inversion qui se développe en surface de la zone $P$ sous la grille. La deuxième - $R_{\mathrm{a}}$ - est la résistance qui traduit l'effet de la couche d'accumulation induite par la polarisation de grille $V_{1}$, à la surface de la région du semiconducteur $\mathrm{N}^{-}$située sous la grille. Cette couche d'accumulation se forme lorsque la tension de drain est inférieure à la tension de grille. La troisième résistance $-R_{\mathrm{d}}-$ est celle de la portion $\mathrm{N}^{-}$du semiconducteur placée entre la couche accumulée sous la grille et le drain $\mathrm{N}^{+}$. En régime de forte accumulation, c'est-à-dire pour les valeurs élevées de la tension grille, la résistance $R_{\mathrm{a}}$ peut être négligée, de sorte que la résistance $R_{\mathrm{ON}}$ s'exprime par :

$$
R_{\mathrm{ON}}=R_{\mathrm{ch}}+R_{\mathrm{d}}
$$

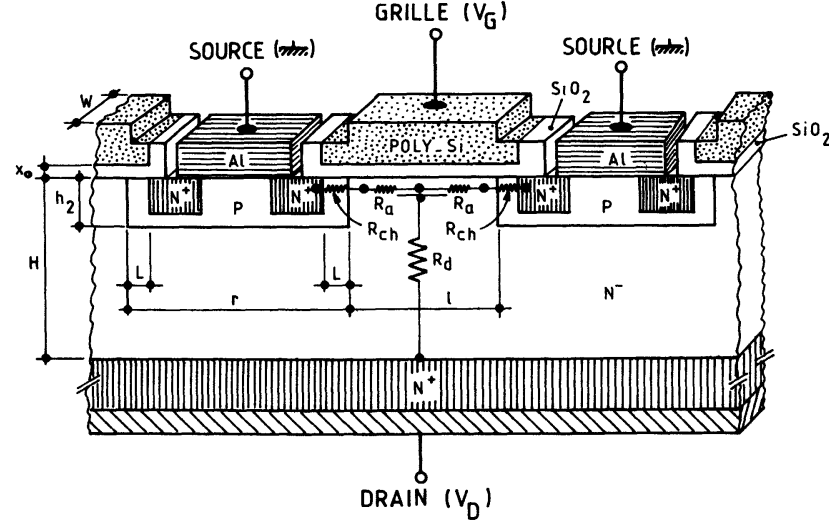

Fig. 3. - Coupe schématique d'une cellule de transistor D.MOS de puissance.

[Cross-section of a power D.MOST cell.]

chacune de ces composantes peut être déterminée en fonction des paramètres géométriques, électriques et technologiques de la structure. La résistance du canal s'exprime par [8] :

$$
\left.R_{\mathrm{ch}}\right|_{V_{\mathrm{D}} \rightarrow 0}=\left(\mu_{0} \frac{Z}{L} \cdot C_{\mathrm{ox}} \cdot \psi\right)^{-1} \cdot \frac{V_{\mathrm{G}}^{\prime}+\psi-2 \varphi_{\mathrm{F}}}{V_{\mathrm{G}}-V_{\mathrm{T}}}
$$

$\mu_{0}$ est la mobilité des porteurs à champ nul, $L$ la longueur des canaux, $C_{\text {ox }}$ la capacité d'oxyde de grille par unité de surface, $\psi$ le potentiel de réduction de mobilité transversal [9], $V_{G}^{\prime}$, la tension effective de grille, $\varphi_{r}$ le potentiel de Fermi de la zone semiconductrice $P$ et $V_{\mathrm{T}}$ la tension de seuil ; $Z$ est la largeur du canal équivalent du transistor MOS de puissance, c'est-à-dire la somme des largeurs $W$ des transistors MOS des cellules élémentaires.

La résistance entre la couche accumulée et la zone $\mathrm{N}^{+}$est essentiellement fonction de la configuration géométrique et de la résistivité de la couche. Les effets de modulation de conductivité qui se manifestent dans les transistors bipolaires, les diodes PIN ou les thyristors, n'apparaissent pas dans le transistor MOS de puissance fonctionnant au voisinage de $V_{\mathrm{D}}$ nul; rappelons toutefois que ce mécanisme de modulation de conductivité est la cause de l'apparition de la région dite de quasi-saturation pour les tensions de drain plus élevees [10]. La résistance normalisée $R_{\mathrm{d}} \cdot W / \rho$, où $W$ est la largeur du canal d'un transistor MOS élémentaire et $\rho$ la résistivité de la couche $\mathrm{N}^{-}$, doit être calculée en toute rigueur en tenant compte des effets de défocalisation des lignes de courant. Elle s'exprime ainsi dans le cas de structures D.MOS (Fig. 3) sous la forme :

$$
\frac{R_{\mathrm{d}} \cdot W}{\rho}=\alpha \cdot \frac{h_{2}}{l} \cdot\left(1-\frac{l}{l+r}\right)+\beta \cdot \frac{H}{l+r}
$$

$H$ est la hauteur de la couche épitaxiée $\mathrm{N}^{-}, h_{2}$ la profondeur de la zone $\mathrm{P}, r$ la longueur de cette zone $\mathrm{P}$, et $l+r$ le pas de répétition de la cellule élémentaire, 
les coefficients $\alpha$ et $\beta$ sont déterminés par intégration bidimensionnelle de l'équation de Laplace dans le semiconducteur $\mathrm{N}^{-}$. Un exemple de résultats ainsi obtenus en utilisant une méthode d'intégration par éléments finis [11] (courbe 1) est représenté sur la figure 4 où l'on a porté les variations de $R_{\mathrm{d}} W / \rho$ en fonction du rapport $H / h_{2}$ lorsque les quantités $l / h_{2}$ et $l /(l+r)$ sont prises comme paramètres.

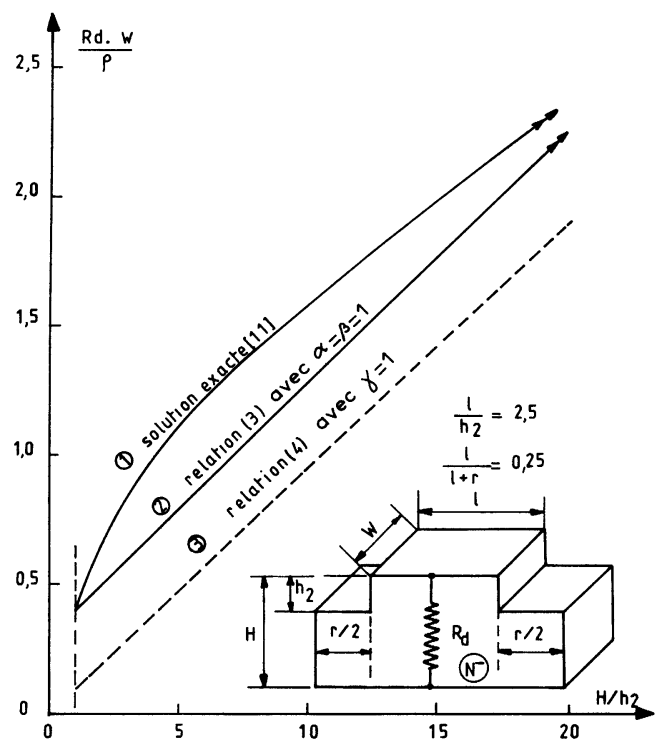

Fig. 4. - Variations de la résistance normalisée $R_{\mathrm{d}} \cdot W / \rho$ en fonction du rapport $H / h_{2}$ : expression exacte et relations approchées.

[Variation of $R_{\mathrm{d}} \cdot W / \rho$ as a function of $H / h_{2}$ : exact and approximate solutions.]

On pourrait admettre en tant que première approximation et en tolérant une erreur de l'ordre de $30 \%$ que, lorsque le rapport $H / h_{2}$ est supérieur à 5 , les coefficients $\alpha$ et $\beta$ peuvent être pris égaux à 1 (courbe 2). Enfin dans le cas de rapports $H / h_{2}$ supérieurs à 10 , ce qui correspond aux cas de transistors dits de moyenne tension, on pourrait admettre en tant que deuxième approximation que la résistance $R_{\mathrm{d}}$ se confond (courbe 3 ) avec celle qu'aurait un barreau semiconducteur de section $W(l+r)$; l'erreur commise ainsi resterait inférieure à $40 \%$. Pour notre part, compte tenu des considérations précédentes nous utiliserons la relation :

$$
\left.\frac{R_{\mathrm{d}} \cdot W}{\rho}\right|_{\| / h_{2} \gg 10} \simeq \gamma \cdot \frac{H}{l+r}
$$

$\gamma$ est un coefficient correctif dépendant de la géométrie, qui varie entre 1 - lorsque $h_{2}$ et $r$ sont nuls et 1,4 ; il est calculable par la méthode des éléments finis.

Compte tenu des relations (2) et (4), la limite asymptotique de la résistance $R_{\mathrm{O}}$, lorsque la tension de grille tend vers l'infini, est prise sous la forme approchée :

$$
R_{\mathrm{O} 、 x} \simeq \frac{1}{\mu_{0} \cdot \frac{Z}{L} \cdot C_{\mathrm{ox}} \cdot \psi}+\gamma \cdot \frac{H}{S} \cdot \frac{1}{q \cdot \mu_{\mathrm{n}} \cdot N_{\mathrm{D}}}
$$

$S$ désigne la surface totale du transistor MOS de puissance, $N_{\mathrm{D}}$ le dopage de la couche $\mathrm{N}^{-}$et $\mu_{\mathrm{n}}$ la mobilité des porteurs dans cette couche; le premier terme représente la résistance du canal et le second terme, la résistance du drain.

2. Le compromis théorique résistance à l'état passant-tension de claquage. - Pour minimiser la contribution de la zone $\mathrm{N}^{-}$à la valeur de la résistance $R_{\mathrm{ON} x}$, on peut diminuer la dimension $H$ et augmenter le dopage $N_{\mathrm{D}}$. Corrélativement ceci entraîne une diminution de la tension de claquage de la diode $\mathrm{P}-\mathrm{N}^{-}$. En considérant en effet que l'avalanche apparaît lorsque le champ au droit de la jonction atteint une certaine valeur critique $E_{\mathrm{B}}\left(E_{\mathrm{B}} \simeq 2 \times 10^{7} \mathrm{~V} / \mathrm{m}\right)$ qui correspond sensiblement à un facteur de multiplication $M$ de 1,01 [11], l'intégration de l'équation de Poisson dans la zone $\mathrm{N}^{-}$conduit à la relation suivante entre la tension de claquage $V_{\text {DBR }}$ et les paramètres de la structure :

$$
V_{\mathrm{DBR}}=\left(\frac{H-h_{2}}{2}\right) \cdot\left(2 E_{\mathrm{B}}-\frac{q \cdot N_{\mathrm{D}} \cdot\left(H-h_{2}\right)}{\varepsilon_{0} \varepsilon_{\mathrm{si}}}\right)
$$

où $\varepsilon_{0}$ est une constante du système MKSA et $\varepsilon_{\mathrm{si}}$ le facteur de permittivité du silicium. Cette tension idéale de claquage est celle que l'on peut atteindre à $5 \%$ près en pratique dans les structures haute tension (200 à $1000 \mathrm{~V})$ par utilisation de technologies spéciales qui suppriment les effets de courbure de jonction [12].

Par élimination de la valeur $N_{\mathrm{D}}$ du dopage entre les relations ( 5 et 6 ), on peut calculer la valeur minimale (pour $H=3 V_{\mathrm{DBR}} / 2 E_{\mathrm{B}}$ ) de la résistance $R_{\mathrm{O} \times x}$ correspondant à une tension de claquage donnée :

$$
\begin{aligned}
R_{\mathrm{ON} \infty \mathrm{MSA}]}= & \left(\mu_{0} \cdot \frac{Z}{L} \cdot C_{\mathrm{ox}} \cdot \psi\right)^{-1}+ \\
& +27 \cdot \gamma \cdot V_{\mathrm{DBR}}^{2} \cdot\left(8 \varepsilon_{0} \varepsilon_{\mathrm{si}} \cdot \mu_{\mathrm{n}} \cdot S \cdot E_{\mathrm{B}}^{3}\right)^{-1}
\end{aligned}
$$

Dans cette évaluation on suppose intrinsèquement que l'épaisseur $h_{2}$ de la couche $P$ est faible devant celle de la zone $\mathrm{N}^{-}$épitaxiée, $H$. On peut noter que, en toute rigueur, il aurait été possible de réduire de $10 \%$ environ le poids du deuxième terme de la relation (7) en considérant une structure à dopage $N_{\mathrm{D}}$ variable avec la profondeur [13]. Cette considération mathématique est trop irréaliste et incompatible avec les techniques d'épitaxie mises en ouvre pour réaliser la couche $\mathrm{N}^{-}$; nous ne l'envisagerons pas dans le cadre de cette étude. Par ailleurs, dans un premier temps, on pourrait modifier légèrement cette relation (7) comme l'indique $\mathrm{C}$. Hu en introduisant (i) la 
loi d'évolution du champ $E_{\mathrm{B}}$ avec la tension de claquage [14]

$$
\underset{[\mathrm{MKSA}]}{E_{\mathrm{B}}}=8,2 \times 10^{7} \cdot V_{\mathrm{DBR}}^{-0,2}
$$

puis (ii) la loi de variation de mobilité des électrons avec le dopage $N_{\mathrm{D}}$ et par suite avec la tension de claquage correspondante [15] $\left(V_{\mathrm{DBR}}>100 \mathrm{~V}\right)$

$$
\begin{aligned}
\mu_{\mathrm{n}} & \simeq 0,07 \times V_{\mathrm{DBR}}^{0,1} \\
\mu_{\mathrm{P}} & \simeq 0,035 \times V_{\mathrm{DBR}}^{0,1} .
\end{aligned}
$$

On obtient ainsi respectivement pour les transistors MOS à canal $\mathbf{N}$ et à canal $\mathbf{P}$ :

$$
\begin{aligned}
& R_{\text {[MKSA] }} \underset{\text { [N }\left.\right|_{N}}{ } \simeq\left(\mu_{0} \cdot \frac{Z}{L} \cdot C_{\mathrm{ox}} \cdot \psi\right)^{-1}+ \\
& +8,3 \times 10^{-13} \cdot \gamma \cdot V_{\mathrm{DBR}}^{2,5} \cdot S^{-1} \\
& R_{\text {[MKSA] }} \simeq\left(\mu_{0} \cdot \frac{Z}{L} \cdot C_{\mathrm{ox}} \cdot \psi\right)^{-1}+ \\
& +16,5 \times 10^{-13} \cdot \gamma \cdot V_{\mathrm{DBR}}^{2,5} \cdot S^{-1} \text {. }
\end{aligned}
$$

Les poids respectifs de chacun des paramètres, dans le cadre de l'évaluation du produit $\left(R_{\mathrm{ON}_{x}} \cdot S\right)^{-1}$ seront analysés dans les deux paragraphes suivants :

2.1 CAS DEs STRUCtures BASSES TENSIONS. Lorsque la tension de claquage est inférieure à $200 \mathrm{~V}$, le premier terme de la relation (10), c'est-à-dire celui qui correspond à la résistance du canal, est prépondérant : le produit $\left(R_{\mathrm{ON} x} \cdot S\right)^{-1}$ est indépendant de la tension de claquage. Il s'exprime par :

$$
\left(\left.R_{\mathrm{ON} \propto}\right|_{\mathrm{N} . \mathrm{P}} \cdot S\right)^{-1}=\left.\mu_{0}\right|_{\mathrm{N} . \mathrm{P}} \cdot \varepsilon_{0} \cdot \varepsilon_{\mathrm{ox}} \cdot \frac{\psi}{x_{0}} \cdot \frac{Z}{S} \cdot \frac{1}{L}
$$

où $\varepsilon_{\mathrm{ox}}$ est le facteur de permittivité de l'oxyde et $x_{0}$ son épaisseur.

Dans l'état actuel de la technologie, il est impossible d'agir sur les paramètres $\mu_{0}, \varepsilon_{\mathrm{ox}}$ et sur le champ critique transversal $\psi / x_{0}$; les deux degrés de liberté que l'on possède sont (i) la longueur $L$ du canal et (ii) le rapport périmètre sur surface $Z / S$. A ce jour les longueurs les plus courtes se situent entre 1 et $4 \mu$.

Le rapport périmètre sur surface $Z / S$ est essentiellement fonction de la densité d'intégration atteinte par le processus technologique utilisé, de la forme géométrique des cellules élémentaires, que sont les diffusions $\mathrm{P}$, et de la distance minimale $l$ à respecter entre ces cellules. La figure 6 représente les évolutions de la quantité $Z . l . S^{-1}$ en fonction du rapport $\pi / l$ lorsqu'on change la forme géométrique (Fig. 5 et table I) de la cellule élémentaire diffusée $\mathbf{P}$ :

a) 1) hexagones inscrits dans un cercle de diamètre $r$,

2) losanges de côté $r$, d'angle $\theta=60^{\circ}$,

b) carrés de côté $r$ alignés,

c) cercles de diamètre $r$ en quinconce,

d) octogones inscrits dans un cercle de diamètre $r$,

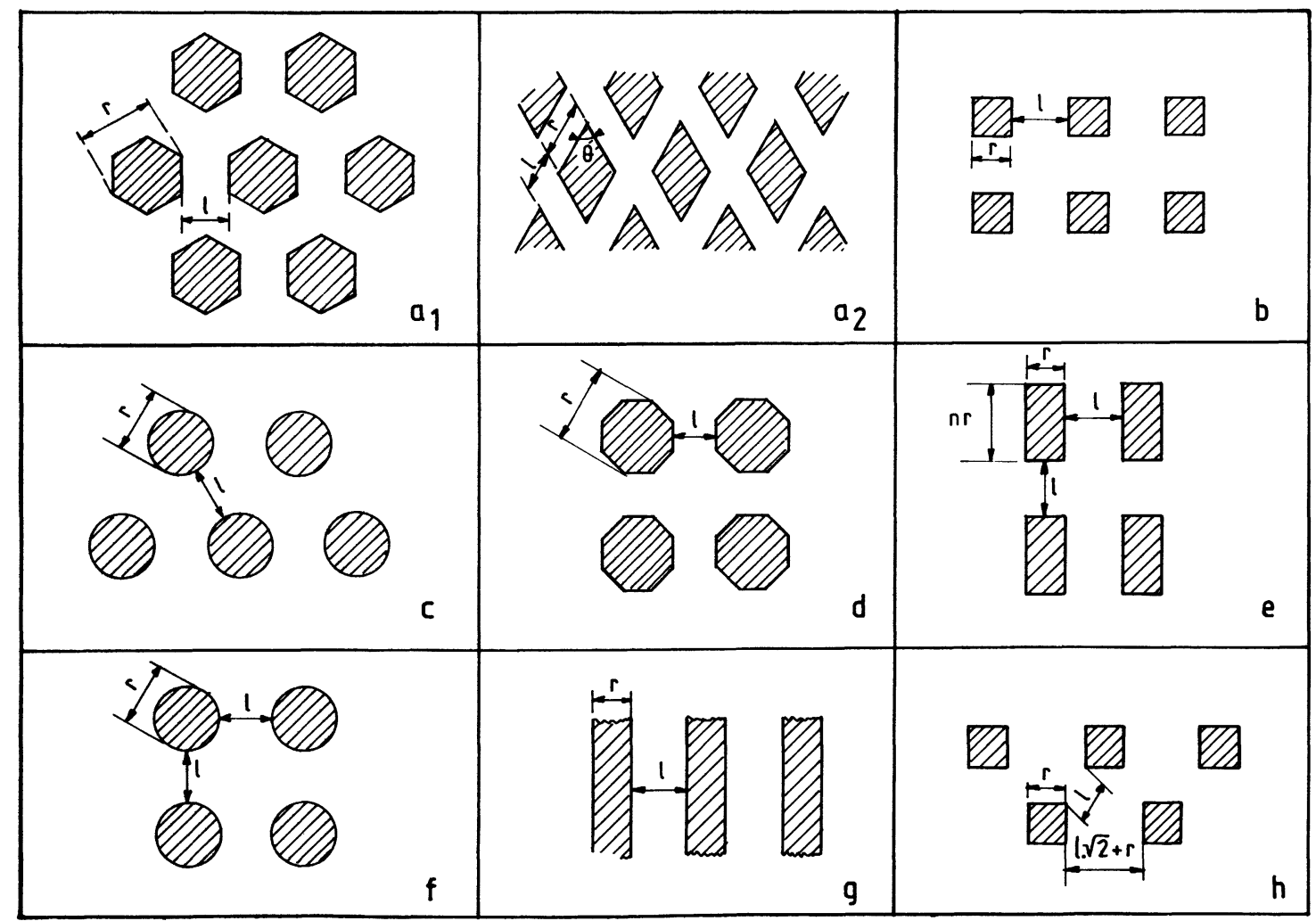

Fig. 5. - Diverses configurations de la diffusion $\mathrm{P}$ de la zone de source.

[Geometrical configurations of the P source diffusions.] 


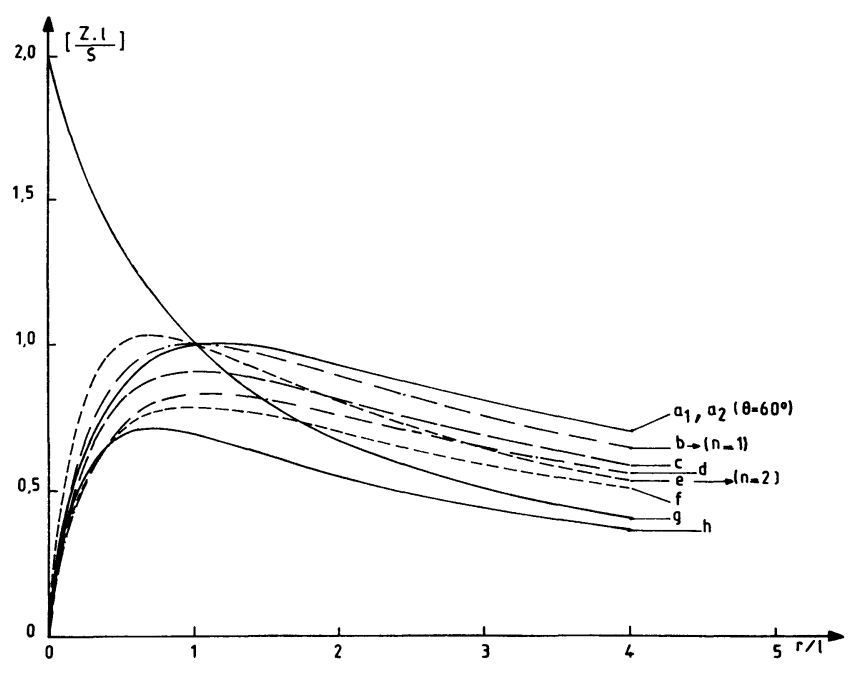

Fig. 6. - Variations de Z.l.S $S^{-1}$ en fonction de $r / l$. Cas des diverses géométries $a_{1}$ : hexagone; $a_{2}$ : losange $\left(\theta=60^{\circ}\right) ; b$ : carrés alignés; c : cercles décalés; $\mathrm{d}$ : octogones; $\mathrm{e}:$ rectangles $(n=2) ; \mathrm{f}:$ cercles alignés; $g$ : bandes parallèles; $h$ : carrés en quinconce.

[Variations of Z.l.S $S^{-1}$ as a function of $r / l$ for various source geometries (corresponding to the figure 5).]

Table I. - Expressions analytiques du produit Z.l.S $S^{-1}$ des motifs géométriques de la figure 5.

[Expressions of the Z.l.S $S^{-1}$ product, corresponding to the geometrical configurations of figure 5.]

\begin{tabular}{|c|c|c|c|}
\hline$a_{1}$ & $2 \sqrt{3} \times \frac{r / 1}{\left(1+\frac{\sqrt{3}}{2} \cdot r / 1\right)^{2}}$ & $d$ & $\frac{8 \sin \frac{\pi}{8} \cdot r / 1}{\left(1+\cos \frac{\pi}{8} \cdot r / 1\right)^{2}}$ \\
\hline$a_{2}$ & $\frac{2}{\cos \frac{\theta}{2} \cdot \sin \frac{\theta}{2} \times \frac{r / 1}{(r / 1+1 / \sin \theta)^{2}}}$ & $f$ & $\frac{\pi \times r / 1}{(1+r / 1)^{2}}$ \\
\hline $\begin{array}{c}b \\
(n=1) \\
e\end{array}$ & $2 \frac{(1+n) \cdot r / 1}{(n)}$ & $g$ & $\frac{2}{1+r / 1) \cdot(1+n r / 1)}$ \\
\hline$c$ & $\frac{2 \pi}{\sqrt{3}} \times \frac{r / 1}{(1+r / 1)^{2}}$ & $h$ & $\frac{1}{n} \cdot \frac{(1+n)\left(1+n^{2}\right) \cdot r / 1}{\left(1+\sqrt{1+n^{2}} \cdot r / 1\right)^{2}}$ \\
\hline
\end{tabular}

e) rectangles de côtés $r$ et $2 r$ alignés,

$f)$ cercles de diamètre $r$ alignés,

$g$ ) bandes parallèles de largeur $r$,

h) carrés de côté $r$ distants de $l$ et de $l \sqrt{2}+r$.

Le cas des rectangles de côtés $r$ et $n r, n$ variant entre 1 et 10 , est représenté sur la figure 7 .

Il apparaît que la quantité Z.l.S ${ }^{-1}$ est affectée par la géométrie des motifs. Le maximum de cette quantité se situe au niveau des structures hexagonales ou carrées au voisinage de $r / l=1$; dans ces conditions Z.l.S $S^{-1}$ est voisin de 1 et le produit $\left(R_{\mathrm{O} .} \cdot S\right)^{-1}$ devient :

$$
\left(R_{\mathrm{ON} x} \cdot S\right)^{-1}=\varepsilon_{0} \varepsilon_{\mathrm{ox}} \cdot \mu_{0} \cdot \frac{\psi}{x_{0}} \cdot(L \cdot l)^{-1}
$$

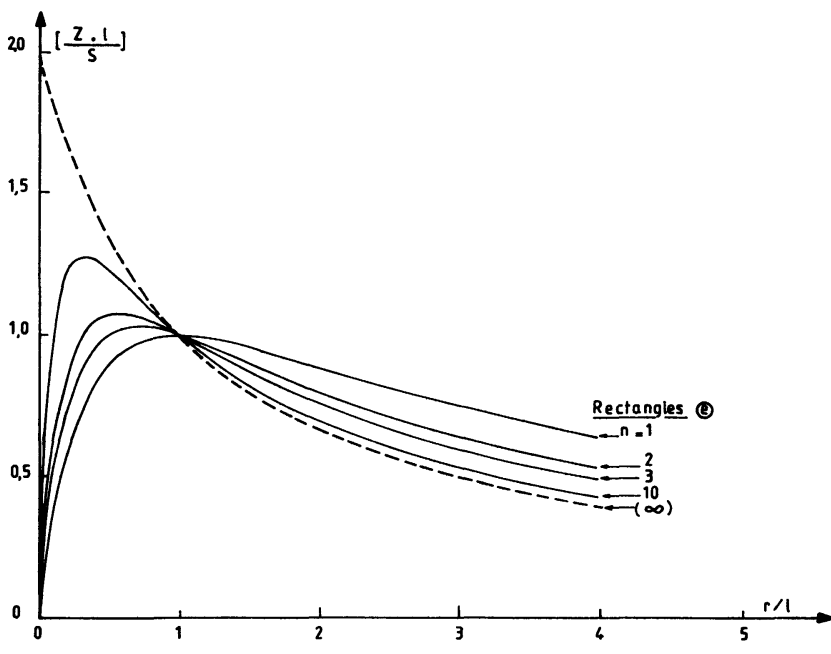

Fig. 7. - Variations de Z.l. $S^{-1}$ en fonction de $r / l$. Cas des rectangles.

[Variations of Z.l.S $S^{-1}$ as a function of $r / l$ for a rectangular source configuration.]

Ainsi le seul degré de liberté restant est le produit de la longueur $L$ de canal par la distance intercellules $l$; nous avons reporté dans le plan $-R_{\mathrm{O} . \mathrm{V}}{ }^{-1}$ par $\mathrm{cm}^{2}$, fonction de $V_{\text {DBR }}-$ (Fig. 8) les variations de la conductance $\mathrm{ON}$ par $\mathrm{cm}^{2}$ lorsqu'on fait varier le produit $l . L$ entre $10 \mu \mathrm{m}^{2}$ et $40 \mu \mathrm{m}^{2}$, les autres paramètres sont fixés à leurs valeurs classiques $\left(\left.\mu_{0}\right|_{\mathrm{v}} \simeq 200 \mathrm{~cm}^{2} / \mathrm{V} / \mathrm{S}\right.$ et $\left.\mu_{0}\right|_{\mathrm{p}} \simeq 150 \mathrm{~cm}^{2} / \mathrm{V} / \mathrm{S}$,

$$
\left.\psi / x_{0}=50 \mathrm{~V} / \mu \mathrm{m}, \quad \text { Z.l. } S^{-1}=1\right)
$$

les caractéristiques obtenues sont horizontales et varient entre $340 \mathrm{~V} / \mathrm{cm}^{2}$ et $85 \mathrm{~V} / \mathrm{cm}^{2}$ (canal N et $L . l=10 \mu \mathrm{m}^{2}$ et $40 \mu \mathrm{m}^{2}$ ).

2.2 CAS Des Structures hautes tensions. Lorsque la tension de claquage est supérieure à $200 \mathrm{~V}$ environ, le poids du deuxième terme, c'est-à-dire celui de la résistance du drain, devient prépondérant dans la relation 10 , de sorte que le produit $\left(S . R_{\mathrm{O} . \mathrm{V}}\right)^{-1}$ est une fonction décroissante - à la puissance -2,5de la tension de claquage :

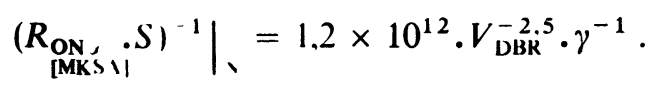

Les variations de la limite théorique maximale de ce produit, obtenues pour $\gamma=1$, sont reportées sur la figure 8 en fonction de la tension $V_{\mathrm{DBR}}$ : la courbe obtenue coupe les trois caractéristiques horizontales précédemment définies, ce qui permet de préciser aux points d'intersection avec ces horizontales, les valeurs des tensions de transition entre les cas que nous avons appelés basses et hautes tensions. Pour les canaux $\mathrm{P}$ les valeurs ainsi obtenues doivent être divisées par 2 puisque la mobilité des trous [9] est la moitié de celle des électrons. 


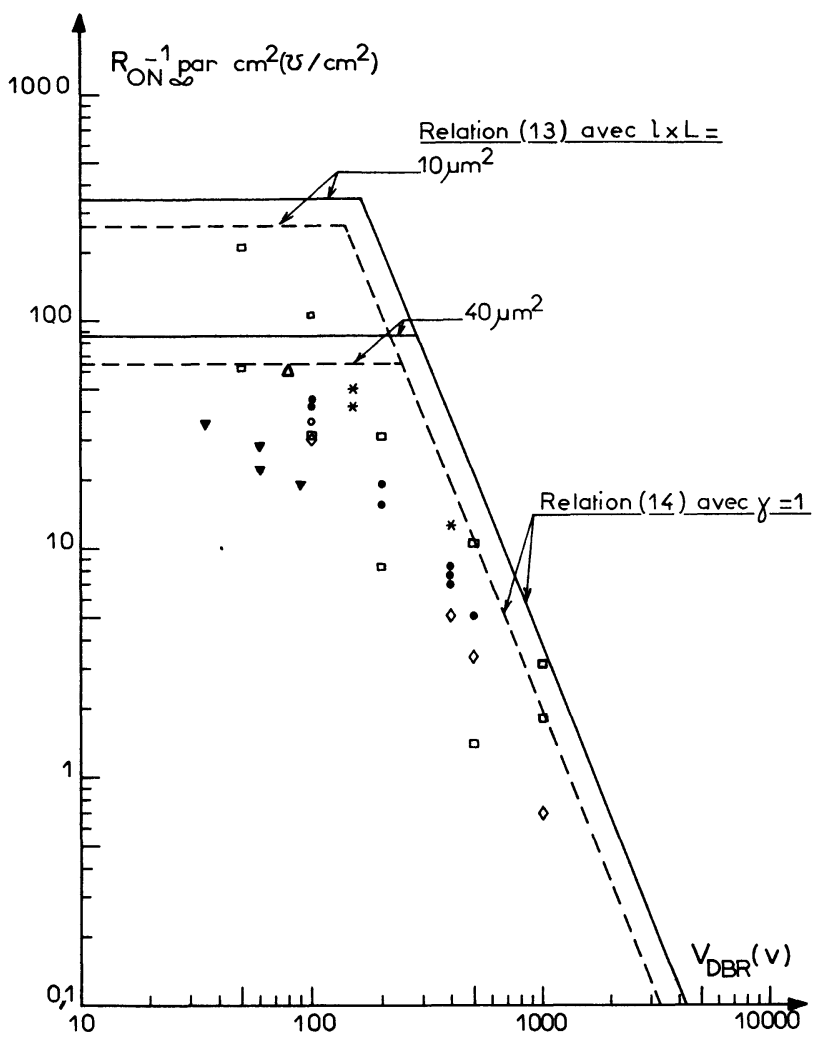

Fig. 8. - Limites théoriques de $\left(R_{\mathrm{ON}} \cdot S\right)^{-1}$ en fonction de $V_{\mathrm{DBR}}$ : - canal $\mathrm{N}$; --.- canal $\mathrm{P}$.

$\underline{\text { Expérience : canaux N }} \quad$ /V.MOS : $\nabla$ non interdigité

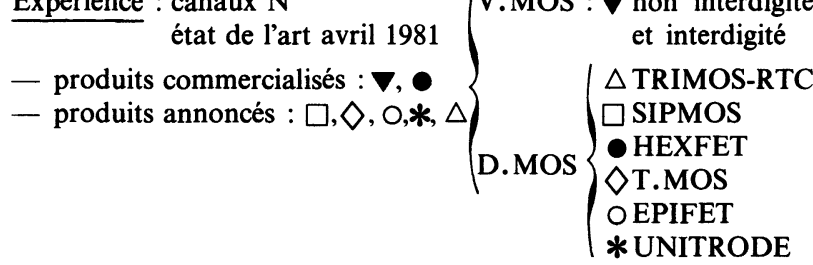

[Theoretical asymptotic values of $\left(R_{\mathrm{ON}} \cdot S\right)^{-1}$ versus $V_{\mathrm{DBR}}$ :

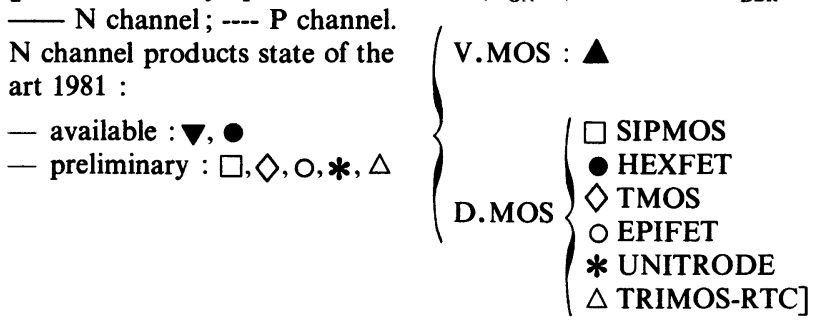

2.3 COMPARAISON AVEC LES RÉSULTATS EXPÉRIMENTAUX. - Les courbes théoriques précédentes peuvent être comparées avec les résultats mesurés sur diverses structures caractéristiques de toutes les familles technologiques développées à ce jour. Ces dernières sont d'une part les SIP MOS et T MOS (source carrée), HEXFET (source hexagonale) et EPIFET (source linéaire) en ce qui concerne les structures D.MOS. Il s'agit d'autre part de la famille V.MOS à sillons linéaires et à source et grille interdigitées [6] ou non interdigitées [8]. On peut constater que tous les points relatifs aux diverses structures se situent en dessous des limites explicitées précédemment. Dans le meilleur des cas, correspondant aux D.MOS, les valeurs expérimentales sont inférieures d'un facteur 3 environ aux valeurs théoriques. Il apparaît donc que l'on peut gagner encore sur le compromis $R_{\mathrm{ON}}^{-1} \cdot S^{-1}$ fonction de $V_{\mathrm{DBR}}$, par des artifices qui sont essentiellement liés aux choix de la structure géométrique et à la technologie de réalisation. Les résultats que l'on obtiendra ainsi pourront représenter une amélioration notable par rapport à ce que l'on sait réaliser aujourd'hui.

3. Conclusion. - Dans cet article, nous avons explicité où se situent les limites extrêmes de la caractéristique $R_{\mathrm{O} . \mathrm{V}}^{-1} \cdot S^{-1}$ en fonction de la tension de claquage $V_{\mathrm{DBR}}$ pour les diverses structures de transistor MOS de puissance; nous avons pris en compte, pour calculer la résistance $R_{\mathrm{O}}$, la part attribuée au canal actif et la part liée à la configuration géométrique de la structure - géométrie de la diffusion de la source $\mathrm{P}$ et du drain $\mathrm{N}^{-}$. Il est apparu que la caractéristique limite $\left(R_{\mathrm{ON}} \cdot S\right)^{-1}$ en fonction de $V_{\mathrm{DBR}}$ peut être représentée en coordonnées logarithmiques par deux droites : l'une horizontale est imposée par les propriétés du canal, et l'autre de pente $-2,5$ est liée au claquage drain-source. Ainsi les structures à canal $\mathbf{N}$ sont meilleures, dans un facteur 2 environ, par rapport aux structures P. De la comparaison entre ces valeurs théoriques et les résultats expérimentaux fournis par toutes les filières technologiques, il apparait que des améliorations sont encore envisageables dans les configurations classiques telles que le D.MOS. Notons enfin que, comparativement aux transistors bipolaires, les transistors MOS présentent un meilleur produit $\left(R_{\mathrm{O} .} . S\right)^{-1}$ jusqu'à des tensions de 300 à $400 \mathrm{~V}$.

Remerciements. - Les auteurs remercient Messieurs J. Tihanyi (Siemens), E. Tonnel (Thomson CSF), J. Lebailly (Radiotechnique), M. Ashikawa (Hitachi) pour tous les renseignements et les structures d'essais qu'ils nous ont fournis. Ils remercient également Mr E. Caquot (CNET Meylan) pour l'aide apportée lors du traitement des équations par la méthode des éléments finis.

\section{ANNEXE}

Cas de V.MOS. - Le calcul de la résistance $R_{\mathrm{d}}$ liée à la présence de la couche épitaxiale $\mathrm{N}^{-}$peut être obtenu dans le cas du V.MOS ou du U.MOS par intégration bidimensionnelle de l'équation de Laplace dans ce semiconducteur [11]. Sur la figure A 1, on a représenté la coupe de la structure $\mathrm{V}$ ou U.MOS; $l$ est l'ouverture en surface et $h^{\prime}$ la profondeur de pénétration de l'attaque anisotrope dans le silicium $\mathrm{N}^{-}, h_{1}$ représente la profondeur la plus grande que l'on puisse obtenir (cas du V.MOS), $H$ est la hauteur de la couche épitaxiale, $\rho$ sa résistivité, et 


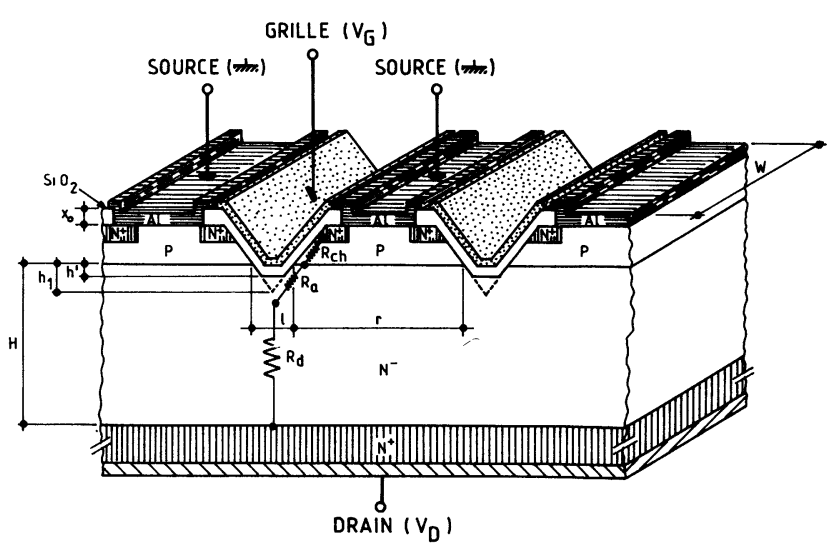

Fig. A1. - Coupe schématique d'une cellule de transistor V.MOS de puissance.

[Cross-section of a V.MOS transistor elementary cell.]

$W$ la largeur du barreau. Le pas de répétition de la structure est égal à $r+l$. Un exemple des résultats obtenus par l'analyse bidimensionnelle est reporté sur la figure A 2 ; on y a tracé les évolutions de $R_{\mathrm{d}} \cdot W / \rho$ en fonction de $H / h^{\prime}$ pour les valeurs des coefficients

$$
h_{1} / l=0,7 \text { et } h_{1} /(l+r)=0,09 .
$$

Le paramètre $h^{\prime} / h_{1}$ évolue entre 1 correspondant au transistor V.MOS et 0,3 pour le U.MOS.

De plus, on a tracé sur la même figure la résistance du barreau massif de section $(r+l) W$ et de hauteur $\left(H-h^{\prime}\right)$ :

$$
\frac{R_{\mathrm{d}} \cdot W}{\rho}=\frac{H-h^{\prime}}{l+r} .
$$

De la comparaison entre cette expression et la solution exacte, il ressort que l'on peut représenter

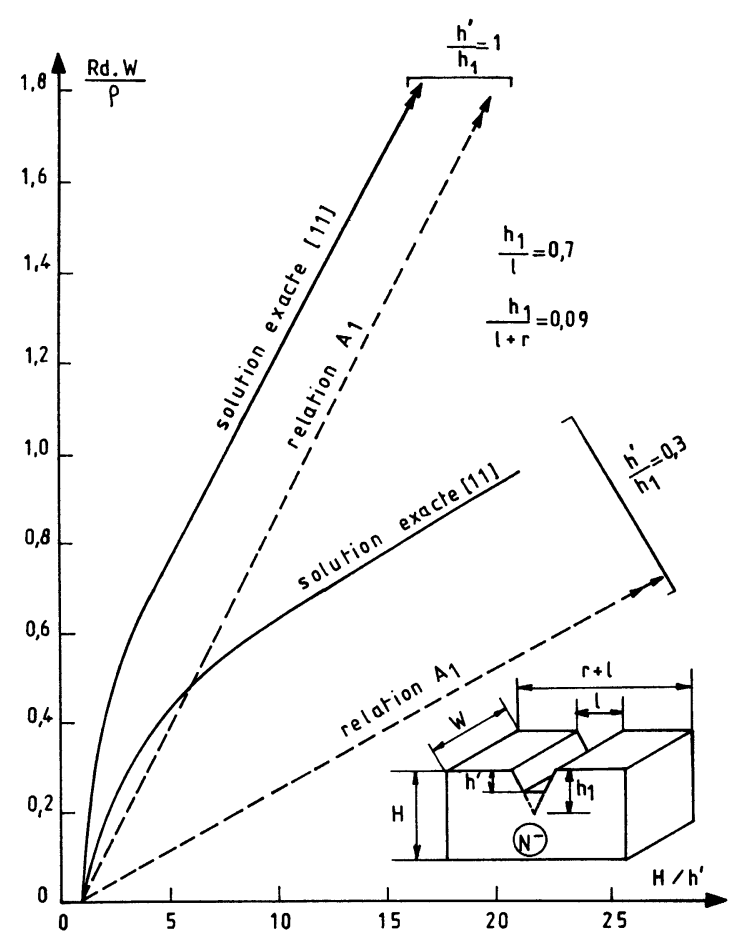

Fig. A2. - Variations de $R_{\mathrm{d}} \cdot W / \rho$ en fonction de $H / h^{\prime}$ dans le cas du transistor V.MOS.

[Variations of $R_{\mathrm{d}} \cdot W / \rho$ as a function of $H / h^{\prime}$ for the V.MOS transistor.]

avec une précision meilleure que $30 \%$, la résistance $R_{\mathrm{d}}$ par la relation approchée A 1. Pour être plus précis on pourrait introduire un terme correctif compris entre 1 et 1,5 qui permet d'exprimer la résistance $R_{\mathrm{d}}$ sous une forme identique à la relation (4) du D.MOS :

$$
\frac{R_{\mathrm{d}} \cdot W}{\rho}=\gamma \cdot \frac{H}{l+r} \text {. }
$$

\section{Bibliographie}

[1] Collins, H. W., Pelly, B., HEXfet, a new power technology, cuts on-resistance, boosts ratings. Electronic Design. Juin (1979) pp. 36-40.

[2] Documentation personnelle fournie par la Société Motorola.

[3] Tihanyi, J., A Qualitative Study of the DC Performance of SIPMOS transistors Siemens R + D. Reports, 4, May (1980).

[4] Documentation personnelle fournie par la Société THOMSONCSF.

[5] OXNer, E., A new technology takes on H.F. Power bipolars. Microwave Systems News Vol. 6 (1976) pp. 107-110.

[6] Salama, C. A. T., A new short channel MOSFET structure (V.MOST). Solid State Electron Vol. 20 (1977) pp. 10031010.

[7] Sun, S. C. and Plummer, J. D., Modeling of the On-Resistance of LDMOS, VDMOS and VMOS Transistors, I.E.E.E. Trans. Electron Devices Vol. ED-27, $\mathrm{n}^{\circ}$ 2, February (1980) pp. 356-367.

[8] Guegan, G., Contribution à l'étude des propriétés dynamiques du transistor Metal-Oxyde-Semiconducteur à canal vertical ( $V M O S)$. Thèse Docteur-Ingénieur, no 663, Université Paul-Sabatier, Toulouse (1979).
[9] VASSILIEFF, G., Modèle du transistor MOS-Influence des variations de la mobilité des porteurs. Thèse $3^{\mathrm{e}}$ cycle, Université Paul-Sabatier, Toulouse (1971).

[10] Caquot, E., Guegan, G., Gamboa, M., Tranduc, H., Rossel, P., Phénomène de Quasi-saturation dans le transistor MOS. Revue Phys. Appl. 15 (1980) pp. 1445-1450.

[11] GambOA, M., Le transistor MOS de puissance; La Relaxation Thermique et les effets liés à la configuration $\mathrm{N}^{-} \mathrm{N}^{+} d u$ drain. Thèse Docteur-Ingénieur, $\mathrm{n}^{\circ} 706$, Université PaulSabatier, Toulouse (1980).

[12] Temple, V. A. K., Love, R. P. and Gray, P. V., A 600 Volt MOSFET designed for Low On-Resistance. I.E.E.E. Trans. Electron Devices Vol. ED-27, $\mathrm{n}^{\circ} 2$ (1980) pp. 343349.

[13] Hu, C., Optimum Doping Profile for Minimum Ohmic Resistance and High-Breakdown Voltage. I.E.E.E. Trans. Electron Devices Vol. ED-26, no 3 (1979) pp. 243-244.

[14] Sze, S. M. and Gibions, G., Avalanche Breakdown Voltages of Abrupt and Linearly Graded P.N. Junctions in Ge, Si, GaAs and GaP. Appl. Phys. Lett. Vol. 8 (1966) pp. 111113.

[15] HU, C., A parametric study of power MOSFETS. Conf. Record, Power Electronics, Specialists Conference, San Diego (1979). 